\title{
Classification Research
}

\section{Global and Local Knowledge Organization, Copenhagen, August 12, 2015}

\author{
Daniel Martínez-Ávila \\ Department of Information Science, São Paulo State University-UNESP, \\ Av. Hygino Muzzi Filho, 737, Marília (17525-900), São Paulo, Brazil, \\ <dmartinezavila@marilia.unesp.br>
}

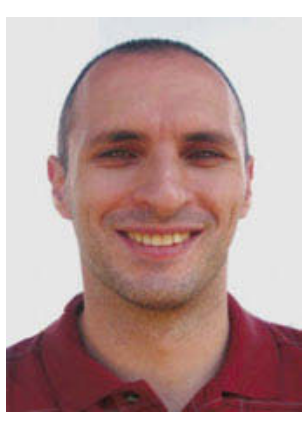

The Global and Local Knowledge Organization conference was held in Copenhagen, Denmark, on August 12, 2015. This one-day conference was chaired by Jens-Erik Mai, University of Copenhagen, Denmark, and coorganized by members from four other countries: José Augusto Chaves Guimarães (São Paulo State University (UNESP), Brazil), Sam Oh (Sungkyunkwan University, Korea), Shigeo Sugimoto (University of Tsukuba, Japan), and Joseph T. Tennis (University of Washington, United States). Delegates from these and other countries (including registered participants from Italy, Sweden and United Kingdom, and distinguished professors such as Richard P. Smiraglia and Birger Hjørland among others) engaged in fruitful conversations on the tension between the global information structures and the meaning and ethics of information in localized contexts, as well as the tension between global and local knowledge organization.

The conference was opened by Jens-Erik Mai, who set out that knowledge organization is shaped in an interdisciplinary spectrum including four lines: information and communication technologies (ICT), theories/philosophy on language and meaning, international standards, and best practices, businesses and organizations. Participants and presentations of the conference would be aligned with these lines while presenting their different points of view and enriching the conversations. Furthermore, Mai established two premises, that $\mathrm{KO}$ is contextual and localized, and that global systems foster international knowledge exchange and communication, and expressed the biggest challenge of this discussion-how to handle localized epistemologies in globalized information infrastructures.

The first of the full presentation was given by José Augusto Chaves Guimarães, titled "Time and Space: Two Axes for Slanted Knowledge Organization.” Continuing with previous studies on bias in knowledge organization in the cultural dimension (e.g., Milani et al. 2014), the author discussed the concepts and differences between bias and slant. In his distinction, slant is "a particular point of view from which something is seen or presented," while bias can be "an attitude, belief, or feeling that results in and helps to justify unfair treatment of a person because of his or her identity" or "an unfair view for or against something," comprising two more negative concepts that were also discussed: prejudice and proselytism. Guimarães advocated for the use of the terms slant and slanted knowledge organization, and presented examples of prejudices in a certain moment that might have been moral values in another period; and virtues in a given social context that might be translated into sins in another context. Finally, he proposed two axes - space and time- (acknowledging inspiration from the two axes of domains for domain analysis proposed by Joe Tennis (2003), and from Tennis's studies on eugenics, e.g., (2012)), to represent the slants in KO procedures, tools and products "in order to contextualize the information content (by recognizing local realities) and to avoid 'universal' categorizations," something that was suggested to be done in "Related Terms" and "Contextual Scope Notes."

The second full presentation was "Library of Congress Classification and cultural imperialism" by Melissa Adler (University of Kentucky, United States). The author followed up her research on bias in Library of Congress Subject Headings, Library of Congress Classification, etc. in relation 
to aspects such as gender and LGBT (see for instance Adler 2009 and 2012) to discuss aspects related to imperialism and colonialism in the LCC. Adler used Foucault to analyze how this bias is created as she discussed how the West is the norm in these systems. She wondered how these projects support the U.S. in a neoliberal, global information society, and argued that "the Library of Congress Classification must be read as part of a history of U.S. nation-building and a technology of cultural imperialism."

The first group of short presentations opened with "Information Cultures: Shaping Information" by Laura Skouvig (University of Copenhagen, Denmark), who examined information from the historical perspective and the historian's point of view. Skouvig pointed out that information is found in all societies, but the way we interact with information is different. Following Neil Postman (1999), she showed how information is not a neutral, objectively existing "thing" but shaped and formed by the context for which it was produced. She discussed information history and genre theory, information culture in the early nineteenth century, the concept of information culture, and the right to information regarding control and surveillance, while illustrating them with examples from the history of Denmark. Fabio Assis Pinho (Federal University of Pernambuco, Brazil) presented "Knowledge Representation of Photographic Documents on Local Systems." He indicated that the use of standards such as General International Standard Archival Description $(I S A D(G))$, International Standard Bibliographic Description (ISBD), Anglo-American Cataloguing Rules 2nd edition (AACR2), Sepiades and Dublin Core for the description of photographic documents might entail problems for information retrieval and the construction of memory, due to the inexistence of fields enabling contextualization. As did Guimarães, he suggested the use of scope notes for a better description of the photographs. Reporting on the results of an exploratory study with a photographic collection at Federal University of Pernambuco, he discussed a way to contextualize photographs in local systems that are integrated in global systems so they can be understood in this global environment. Pinho concluded that "for the application of documentary analysis to the photographic collection it was also necessary to consult other documents for the contextualization of the photography and thus contribute to the construction of memory." Finally, Karolina Lindh (Lund University, Sweden) presented "Breathing Life into a Standard: Turning Universal Resuscitation Guidelines into Embodied Know-how." She drew on Bowker and Star (1999) and Star and Lampland (2009) to analyze cardiopulmonary resuscitation (CPR) standards and CPR activities in light of how standards shape activities and become invisible as they are integrated across practices. This study showed how something that is supposed to be general and universal, such as the standardized CPR procedure, is transformed in situated local enactments.

In the following full presentation, Shigeo Sugimoto (University of Tsukuba, Japan) talked on "Metadata for Manga: Metadata Vocabulary, Schema and Tools." Sugimoto introduced manga to the audience as well as the current metadata projects at the University of Tsukuba, such as the Manga Metadata Framework based on Functional Requirements for Bibiliographic Records (FRBR) (see Morozumi et al. 2009), the discussion and identification of works and other FRBR entities in this model, and the software tools to help access to and creation of manga in the digital environment. Sugimoto mainly focused on the technological aspects of metadata for manga, reviewing the various resources related to a work of manga, highlighting the use of Wikipedia as a crowd authority, and explaining the characteristics of the metadata in the various stages of the production process. Finally, the author noted the use and importance of ontologies as a basis for the share of metadata on the Internet and to enhance interoperability across communities.

Sam Oh, Professor (Sungkyunkwan University, Korea) gave another full presentation entitled "A Role of Ontology in Social Data Analytics" on the use of ontology in text data mining. He presented a project with LG-CNS (a leading advanced analytics company in Korea) that seeks to improve sentiment analysis (also known as opinion mining) by modeling vocabulary with SKOS Simple Knowledge Organization System and Web Ontology Language and Resource Description Framework (RDF/OWL). Oh reported on the construction and visualizing of the smartphone ontology and discussed the benefits of the ontology in social data analytics. In this project, the advantages of the types of visualization and advantages were evaluated by experts in social data analytics and experts and experts in social marketing.

The next presentation was "Meaning-making in Global and Local Information Infrastructures: an Argument Coming from Genre Theory" by Jack Andersen (University of Copenhagen, Denmark). Andersen discussed meaning-making and genre theory in global and local information infrastructures. He stated that "in the contemporary digital information society almost all communication and interaction is shaped and guided by structures designed and constructed by information professionals trained in knowledge organization," emphasizing the inextricable intertwining of $\mathrm{KO}$ with media and communication. Then he addressed concepts such as meaning, 
communication and interaction, digital media and its genres, including search engines, social-, mass-, and networked-media, and genre and activity systems at global and local levels. The author showed how contexts that are considered global are only global to a certain extent and that something can be global and local at the same time. Andersen's presentation was followed by a very interesting conversation about Google and the role of knowledge organizers in this new scenario and how Google would be there with or without $\mathrm{KO}$.

The following block opened with "Reader-interest Classifications: Local Classifications or Global Industry Interest?" by Daniel Martínez-Ávila (São Paulo State University, Brazil). This presentation dealt with the tensions between rigid standardization and local interests in library classifications, exemplified by the cases of reader-interest classifications and bookstore classifications in libraries. Following up previous studies on the topic (e.g., Martínez-Ávila et al. 2012; Martínez-Ávila et al. 2014), issues such as the role of standardization and centralization in these projects, the focus and philosophy underlying the construction of these classifications, and the underlying global interests of the book industry were discussed to determine the independence and viability of these classifications. Discussions on aspects such as the oxymoron of OCLC's WorldCat (Fox and Olson 2012), and the concept of market warrant (Martínez-Ávila and Kipp 2014) were some of the highlights of the presentation. The next short presentation was given by Tsunagu Honma (University of Tsukuba, Japan), entitled "Organizing Existing Metadata Terms and Structural Constraints to Support Metadata Schema Creation." Honma examined aspects related to metadata schema creation for linked open data datasets and on how to provide infrastructure to support finding and choosing metadata terms and reusing existing terms to improve interoperability of datasets. Finally, Ole Olesen-Bagneux (University of Copenhagen, Denmark) spoke on "The Post-global Internet." He reviewed the different initiatives to resist and challenge the dominance of the U.S.-dominated Internet: from the early attempts of International Standards Organization (ISO) standardized protocols instead of the Internet Protocols (IP) to the competing structures of satellite and cable distribution of the BRICS (Brazil, Russia, India, China and South Africa). In his words: "The internet was at extreme pace linking the entire planet together in a communication platform that was also the archive of the communication platform. Everything could be said, shared and stored, on a global scale."

Closing the full presentations, Melanie Feinberg (University of North Carolina at Chapel Hill), proceeded with "A Third Kind of Power: KO for Interpretive Mastery." She applied and expanded Patrick Wilson's (1968) concepts of exploitative control and descriptive control with examples from maps, databases and search engines such as Google, Google Scholar and PubMed to propose a third kind of power: exploitative control. Feinberg commented on a paper by Birger Hjørland (2015) in which it is stated that "Boolean searching facilitates mastery over a system- Boolean results can be interpreted by the searcher in a way that facilitates interpretive control over the system." She pointed out that even if systems provided perfect exploitative control they should include mechanisms for interaction of the users too. Thus mastery of the databases is important to achieve better results. She concluded that interpretive control enables users to generate knowledge from information systems (not just documents) and $\mathrm{KO}$ enables that interpretive control.

For the concluding remarks, ISKO President Joseph Tennis (University of Washington, United States) talked on "Local and Global Knowledge Organization: Constructs, Commonalities, and our New Cartography.” Drawing from the presentations, he listed some constructs related to the global, the standards, and the local, inferred some commonalities among the presentations, and wondered what our new cartography should be, pointing out perhaps the need of more case studies in relation to the global and the local. Finally, he referred to some of the thoughts of the audience including looking at media, global and local in other frames (e.g., religion, colonization, economics, etc.), a global commitment with information cultures, watching $\mathrm{KO}$ activities, among others.

\section{References}

Adler, Melissa. 2009. "Transcending Library Catalogs: A Comparative Study of Controlled Terms in LCSH and User-Generated Tags in LibraryThing for Transgender Books." Journal of Web Librarianship 3: 309-31.

Adler, Melissa. 2012. "Disciplining Scholarship at the Library of Congress." Knowledge Organization 39: 370-6.

Bowker, Geoffrey C. and Susan Leigh Star. 1999. Sorting Things Out: Classification and its Consequences. Cambridge, Mass : MIT Press.

Fox, Melodie and Hope A. Olson. 2012. "Feminist Epistemologies and Knowledge Organization." In Cultural Frames of Knowledge, edited by Richard P. Smiraglia and Hur-Li Lee. Würzburg, Germany: Ergon Verlag, 79-97.

Hjørland, Birger. 2015. "Classical Databases and Knowledge Organization: A Case for Boolean Retrieval and Human Decision-Making During Searches." Journal of the Association for Information Science and Technology 66: 1559-75. 
Martínez-Ávila, Daniel and Margaret Kipp. 2014. "Implications of the Adoption of BISAC for Classifying Library Collections." Knowledge Organization 41: 377-92.

Martínez-Ávila, Daniel, Margaret Kipp and Hope A. Olson. 2012. "DDC or BISAC: The Changing Balance Between Corporations and Public Institutions." Knowledge Organization 39: 309-19.

Martínez-Ávila, Daniel, Rosa San Segundo and Hope A. Olson. 2014. "The Use of BISAC in Libraries as New Cases of Reader-Interest Classifications.” Cataloging \& Classification Quarterly 52 no. 2: 137-55.

Milani, Suellen Oliveira, José Augusto Chaves Guimarães and Hope A. Olson. 2014. "Bias in Subject Representation: Convergences and Divergences in the International Literature." In Knowledge Organization in the 21st Century: Between Historical Patterns and Future Prospects: Proceedings of the Thirteenth International ISKO Conference, 19-22 May 2014, Krakón, Poland, edited by Wieslaw Babik. Advances in Knowledge Organization 14. Würzburg: Ergon Verlag, 96-100.

Morozumi, Ayako, Satomi Nomura, Mitsuharu Nagamori and Shigeo Sugimoto. 2009 "Metadata Framework for Manga: A Multi-paradigm Metadata Description
Framework for Digital Comics." In Proceedings of International Conference on Dublin Core and Metadata Applications 2009, Seoul: Dublin Core Metadata Initiative; Korean Library Association, 61-70.

Postman, Neil. 1999. Building a Bridge to the 18th Century: How the Past Can Improve Our Future. New York: Alfred A. Knopf.

Star, Susan Leigh and Martha Lampland. 2009. "Reckoning with Standards." In Standards and their Stories: How Quantification, Classifying and Formalization Practices Shape Everyday Life, edited by Martha Lampland and Susan Leigh Star. Ithaca: Cornell University Press, 3-24.

Tennis, Joseph T. 2003. "Two Axes of Domains for Domain Analysis." Knowledge Organization 30: 191-5.

Tennis, Joseph T. 2012. “The Strange Case of Eugenics: A Subject's Ontogeny in a Long-Lived Classification Scheme and the Question of Collocative Integrity." Journal of the American Society for Information Science and Technology 63: 1350-9.

Wilson, Patrick. 1968. Two Kinds of Power: An Essay on Bibliographical Control. Berkeley: University of California Press. 